\title{
Antimicrobial resistance of Klebsiella Pneumoniae Pathogens in 2019 of Shaoxing City, Zhejiang Province: A Multi-Centre, Retrospective Study
}

\author{
Meichun Liang \\ Shaoxing People's Hospital \\ Guofeng Mao \\ Shaoxing People's Hospital \\ Xiaojiao Zhang \\ Shaoxing People's Hospital \\ Qiuli He \\ Shaoxing People's Hospital \\ Qunhua Ying
}

Shaoxing Maternity and Childcare Hospital: Shaoxing Women and Children's Hospital

\section{Sheliang Wang}

Shaoxing Second Hospital

\section{Faxiang Jin}

Shaoxing University Affiliated Hospital

\section{Su Dong}

Shaoxing Traditional Chinense Medicine Hospital

Xiuqin Lin

Shaoxing Seventh Hospital

\section{Yongchun Ruan}

Shaoxing People's Hospital

\section{Minghui Li}

Shaoxing People's Hospital

Li Lv

Shaoxing People's Hospital

Yiqing Zhou ( $\triangle$ chyq1012@126.com )

Shaoxing People's Hospital https://orcid.org/0000-0002-5286-7939

\section{Research article}

Keywords: Klebsiella pneumoniae, antibiotic resistance, ESBL, CRKP, Epidemiology

Posted Date: December 22nd, 2020

DOI: https://doi.org/10.21203/rs.3.rs-132287/v1 
License: (-) (1) This work is licensed under a Creative Commons Attribution 4.0 International License. Read Full License 


\section{Abstract}

Background: The detection rate and drug resistance rate of $K$. pneumoniae increased year by year in China. Understanding the drug resistance situation will help guide prevention and control of K. pneumoniae infection.

Methods: This study collected the results of drug susceptibility of $K$. pneumoniae from six tertiary hospitals in Shaoxing city in 2019 and analyzed the differences in drug resistance among different hospitals, genders, ages, and specimens.

Results: A total of 1954 strains were collected, all of which were most sensitive to amikacin, with a resistance rate of $4.42 \%$. The resistance rate to carbapenems and tigecycline was less than $10 \%$. Nitrofurantoin had the highest resistance rate (36.18\%). The drug resistance rate of Shaoxing traditional Chinese medicine hospital and Shaoxing maternity and child health care hospital was lower, and the drug resistance rate of the affiliated hospital of Shaoxing University was the highest. In addition to the Shaoxing maternity and child health care hospital, specimens were mainly from elderly and male patients, the drug resistance rate increased with age, and no carbapenem-resistant strains were found in patients under 17 years old. The specimens were mostly from the respiratory tract and urinary tract, but the CRKP rate was the highest in blood, the ESBL rate was the highest in other sterile body fluids except for blood, and the high ESBL and CR rates in urine were also detected.

Conclusions: The above results indicated that there were significant differences in drug resistance rates of strains among different hospitals, genders, ages, and specimen sources, and clinical empirical medication should be incorporated into the reference.

\section{Introduction}

Klebsiella pneumoniae is one of the most important and challenging pathogens from the community- and hospital-acquired infections, as it expresses multidrug resistance (MDR) phenotypes and causes infections with high mortality. Carbapenems are mainly used to treat strains that produce extended-spectrum $\beta$-lactamases, but the resistance rate of $K$. pneumoniae is increasing year by year. According to data from the China Carbapenemresistant Enterobacteriaceae (CRE) Network, 73.9\% of CRE strains are Carbapenem-resistant K. pneumoniae (CRKP) [1]. The resistance rate of $K$. pneumoniae to imipenem and meropenem fluctuated around $18.6 \%$ and $64.1 \%$ by China antimicrobial surveillance network(CHINET)[2]. CRKP, Carbapenem-resistant $P$. aeruginosa (CRPA), and Carbapenem-resistant $A$. baumannii(CRAB) were recognized as critical priority pathogens by the World Health Organization (WHO)[3]. Among them, CRKP had the larger impact on economic cost and mortality rate by a China multi-center study [4].

A critical trait of $K$. pneumoniae is its ongoing evolution by acquiring new mobile genetic elements associated with antimicrobial resistance[5]. The most problematic are genes that encode extended-spectrum $\beta$-lactamases (ESBLs) (e.g., SHV, CTX-M, and TEM) and carbapenemases (KPC, NDM, IMP, OXA, etc). Among them, KPC-2 is the most important carbapenemase, in addition to NDM-1, OXA-48-like carbapenemases, etc[6].

The distribution and antibiotic susceptibilities of $K$. pneumoniae vary in different regions and hospitals. Empiric antibiotic treatments should be selected based on susceptibility data. We conducted drug resistance monitoring of K. pneumoniae clinically isolated from six hospitals in Shaoxing City from January 1 to December 31, 2019, to guide the application of empirical antibiotics. 


\section{Materials And Methods \\ Sample size and source}

Six tertiary hospitals in Shaoxing, Zhejiang province, were investigated in 2019. There are three general hospitals(Shaoxing people's hospital(SPH), The affiliated hospital of Shaoxing University(SUH), Shaoxing second hospital(2nd H)), and three specialist hospitals (Shaoxing traditional Chinese medicine hospital(TCM), Shaoxing maternity and child health care hospital(SMCH), Shaoxing seventh hospital(7th $\mathrm{H})$ ). A total of $1954 \mathrm{~K}$. pneumoniae strains were collected. 870 strains were isolated from Shaoxing people's hospital, 289 from Shaoxing second hospital, 289 from Shaoxing maternity and child health care hospital, 261 from the affiliated hospital of Shaoxing University, 166 from traditional Chinese medicine hospital, and 79 from Shaoxing seventh hospitals. There were 1,149 strains from male patients and 805 strains from female patients. Age from 1 day to 99 years, divided into three groups: $\leqq 17$ years, $18-64$ years, $\geqq 65$ years.

The main sources of specimens include respiratory specimens, urine, blood, sterile body fluids, and non-sterile body fluids. The sterile body fluids include bile, cerebrospinal fluid, ascites, pleural fluid, etc. Non-sterile body fluids include wound secretions, lochia, cervical secretions, drainage, pus, etc.

\section{Antimicrobial susceptibility testing}

Isolates identification and antibiotic susceptibility testing were carried out using an automated Vitek-2 compact system by bioMerieux company France. K-B disk method was used for meinopenan, ceftazidime, cefoperazone/sulbactam. In this study, the susceptibility interpretations are based on CLSI clinical breakpoints[7]. Carbapenem-Resistant $K$. pneumoniae (CRKP) was defined according to the recommendation of $\mathrm{CLSI}$ as $K$. pneumoniae strains resistant to at least one carbapenem agent such as ertapenem, imipenem, or meropenem[8]. Extended-spectrum $\beta$-lactamase K. pneumoniae (ESBL-KP) strain was determined by the standard double-disc synergy test with cefotaxime (30 ug) and ceftazidime (30 ug) alone and in combination with clavulanic acid (10 ug; Oxoid Limited, UK) as recommended by CLSI. The E. coli American Type Culture Collection (ATCC) 25922(negative control) and K. pneumoniae ATCC 700603 (positive ESBL control) were used as quality control (QC) strains. Data were only included when the QC test results were in acceptable ranges.

\section{Statistical analysis}

Data collected by WHONET5.6 software. The SPSS 17.0 statistical software was used for data analysis after the data were exported and verified. Counting data are expressed in percentages. The $\chi 2$ test was used to assess the differences with data comparison. The statistical significance level was set at $p<0.05$.

\section{Results}

\section{Distribution of K. pneumoniae strains}

Among the 1954 isolated strains, $\leqq 17$ years, $18-64$ years, $\geqq 65$ years of patients accounted for $5.32 \%, 41.10 \%$, $53.58 \%$, respectively. Except for Shaoxing maternity and child health care hospital, the detection rate of men was higher than that of women. The detection rate of men in TCM hospital was the highest which is $71.08 \% . K$. pneumoniae isolated from respiratory tract samples such as sputum accounted for $52.61 \%$, K. pneumoniae isolated from non-sterile body fluids such as secretions is $15.56 \%$, sterile body fluids such as urine, bile, and 
blood accounted for $15.51 \%, 11.82 \%, 4.50 \%$,respectively. The detection rate of $K$. pneumoniae in $\leqq 17 y$ group in the Shaoxing maternity and child health care hospital was $33.22 \%$, and we didn't isolate strains in elderly patients and the blood samples. The detection rate of $K$. pneumoniae in non-sterile body fluids was $67.13 \%$, of which mainly vaginal secretions (61.94\%), as shown in Table 1.

Table 1

The detection rate (\%)of $K$. pneumoniae strains in various hospitals

\begin{tabular}{|c|c|c|c|c|c|c|c|c|c|c|}
\hline Hospital & $\mathrm{Age}^{\mathrm{a}}$ & Male & $\leqq$ & 18囚64y & $\geqq_{65 y}$ & $\begin{array}{l}\text { Respiratory } \\
\text { tract } \\
\text { samples }\end{array}$ & Urine & Blood & $\begin{array}{l}\text { Sterile } \\
\text { body } \\
\text { fluids }\end{array}$ & $\begin{array}{l}\text { Non- } \\
\text { sterile } \\
\text { body } \\
\text { fluids }\end{array}$ \\
\hline SPH & $\begin{array}{l}66.79 \\
\pm \\
14.75\end{array}$ & 68.74 & 0.69 & 38.16 & 61.15 & 62.76 & 12.76 & 4.37 & 9.31 & 10.80 \\
\hline 2ndH & $\begin{array}{l}65.62 \\
\pm \\
17.87\end{array}$ & 61.59 & 0.69 & 38.75 & 60.55 & 33.91 & 22.84 & 9.00 & 5.88 & 28.37 \\
\hline SUH & $\begin{array}{l}70.68 \\
\pm \\
16.29\end{array}$ & 63.60 & 0.00 & 35.63 & 64.37 & 54.79 & 22.99 & 5.75 & 6.90 & 9.58 \\
\hline TCM & $\begin{array}{l}68.67 \\
\pm \\
16.56\end{array}$ & 71.08 & 0.00 & 35.54 & 64.46 & 77.71 & 4.82 & 4.82 & 0 & 12.65 \\
\hline 7thH & $\begin{array}{l}75.65 \\
\pm \\
15.13\end{array}$ & 50.63 & 0.00 & 17.72 & 82.28 & 69.62 & 27.85 & 1.27 & 0.0 & 1.27 \\
\hline SMCH & $\begin{array}{l}27.51 \\
\pm \\
21.83\end{array}$ & 16.96 & 33.22 & 66.78 & 0.00 & 19.72 & 12.46 & 0.00 & 0.35 & 67.47 \\
\hline Mean & $\begin{array}{l}61.85 \\
\pm \\
22.17\end{array}$ & 58.80 & 5.32 & 41.10 & 53.58 & 52.61 & 15.51 & 4.50 & 5.99 & 21.39 \\
\hline
\end{tabular}

1. K. pneumoniae drug sensitivity results in various hospitals

K. pneumoniae is the most sensitive to amikacin, with a resistance rate of $4.42 \%$, resistance to carbapenem antibiotics and tigecycline less than $10 \%$, and the average resistance rate to $\beta$-lactam- $\beta$-lactamase inhibitor combinations ranging from 9.95 to $16.92 \%$. The resistance rate of cephalosporin, aztreonam, cefoxitin, ciprofloxacin, nitrofurantoin, and Sulfamethoxazole is $12.63-28.72 \%$. Nitrofurantoin (36.18\%) had the highest drug resistance rate in the Shaoxing people's hospital, and the other antibiotics had the highest drug resistance rate in the affiliated hospital of Shaoxing University. There are significant differences in the resistance rate between hospitals $(p<0.05)$, as shown in Table 2. 
Table 2

Comparison of the antibiotic resistance rate (\%) of $K$. pneumoniae in various hospitals in Shaoxing city

\begin{tabular}{|llllllllll|}
\hline Antibiotics & SPH & 2ndH & SUH & TCM & 7thH & SMCH & Mean & X2 & $\boldsymbol{p}$ \\
\hline Ampicillin & 99.75 & 83.39 & 88.51 & 100.00 & 79.75 & 69.55 & 87.67 & 174.76 & 0.00 \\
\hline Cefazolin & 30.13 & 33.91 & ND & 15.00 & 29.11 & 23.18 & 28.42 & 14.06 & 0.01 \\
\hline Ceftazidime & 17.36 & 18.40 & 32.95 & ND & ND & ND & 20.45 & 30.88 & 0.00 \\
\hline Ceftriaxone & 26.67 & 31.14 & 47.13 & 13.25 & 27.85 & 17.65 & 27.65 & 83.32 & 0.00 \\
\hline Cefepime & 16.80 & 12.80 & 31.03 & 3.61 & 10.13 & 1.38 & 14.44 & 119.54 & 0.00 \\
\hline Amoxicillin / Clavulanic & 18.80 & 33.33 & ND & 5.42 & 13.92 & 4.15 & 13.95 & 51.74 & 0.00 \\
acid & & & & & & & & & \\
\hline Piperacillin/Tazobactam & 9.68 & 13.59 & 22.22 & 3.61 & 8.86 & 0.00 & 9.95 & 87.67 & 0.00 \\
\hline Cefoperazone/sulbactam & 13.33 & 92.68 & ND & ND & ND & ND & 16.92 & 175.31 & 0.00 \\
\hline Aztreonam & 22.70 & 26.99 & 40.23 & 8.43 & 20.25 & 9.69 & 22.36 & 97.07 & 0.00 \\
\hline Cefoxitin & 15.71 & 16.67 & ND & 4.82 & 18.99 & 6.23 & 12.63 & 30.21 & 0.00 \\
\hline Amikacin & 4.26 & 6.01 & 9.58 & 1.20 & 6.33 & 0.00 & 4.42 & 36.31 & 0.00 \\
\hline Gentamicin & 15.50 & 15.22 & 28.74 & 6.02 & 8.86 & 14.88 & 16.24 & 46.63 & 0.00 \\
\hline Ciprofloxacin & 24.05 & 33.56 & 47.89 & 15.06 & 27.85 & 21.45 & 28.72 & 77.27 & 0.00 \\
\hline Levofloxacin & 18.89 & 17.30 & 37.16 & 9.04 & 18.99 & 9.69 & 18.90 & 83.81 & 0.00 \\
\hline Macrodantin & 36.18 & 23.18 & 34.10 & 31.33 & 26.58 & 12.46 & 27.60 & 57.37 & 0.00 \\
\hline Sulfamethoxazole & 24.42 & 30.21 & 30.27 & 10.98 & 25.32 & 23.53 & 24.83 & 25.32 & 0.00 \\
\hline Tobramycin & 9.29 & 9.34 & 16.48 & 5.42 & 8.86 & 4.50 & 9.18 & 27.11 & 0.00 \\
\hline Ertapenem & 7.76 & 17.36 & 23.37 & 1.23 & 0.00 & 0.69 & 9.42 & 129.43 & 0.00 \\
\hline Imipenem & 7.02 & 16.61 & 21.46 & 1.83 & 8.86 & 0.00 & 8.97 & 113.26 & 0.00 \\
\hline Meropenem & 6.83 & ND & ND & ND & ND & ND & 6.83 & - & - \\
\hline Tigecycline & 8.65 & 16.67 & ND & 0.64 & 0.00 & 1.04 & 5.76 & 38.32 & 0.00 \\
\hline ND: not determined & & & & & & & & & \\
\hline
\end{tabular}

\section{K. pneumoniae drug sensitivity results in different people groups}

Among isolated strains in the $\leqq 17 y$ group, no carbapenem-resistant strains were detected, and no strains resistant to amikacin, piperacillin/tazobactam, cefoperazone/sulbactam. The drug resistance rate of cefazolin in the $\leqq 17 y$ group was $36.63 \%$, which was significantly higher than that of other age groups $(x 2=23.67, p=0.00)$. For other antibiotics, the drug resistance rate of the $\geqq 65 y$ group was the highest(Table 3$)$. Except for cefoperazone and compound trimethoprim, the resistance rate of other commonly used antibiotics derived from male patients was significantly higher than that from female patients(Table 4). 
Table 3

Comparison of antibiotic resistance rates (\%) of $K$. pneumoniae from different age groups

\begin{tabular}{|llllll|}
\hline Antibiotics & $\leqq 17 y$ & $18 \times 64 y$ & $\geqq 65 y$ & $\chi 2$ & $p$ \\
\hline Ampicillin & 70.30 & 83.71 & 93.18 & 58.66 & 0.00 \\
\hline Cefazolin & 36.63 & 20.95 & 33.65 & 23.67 & 0.00 \\
\hline Ceftazidime & 12.50 & 13.43 & 24.86 & 26.90 & 0.00 \\
\hline Ceftriaxone & 22.12 & 19.42 & 34.49 & 52.72 & 0.00 \\
\hline Cefepime & 1.92 & 9.09 & 19.79 & 56.02 & 0.00 \\
\hline Amoxicillin / Clavulanic acid & 8.82 & 8.47 & 19.31 & 33.73 & 0.00 \\
\hline Piperacillin/Tazobactam & 0.00 & 5.36 & 14.46 & 54.08 & 0.00 \\
\hline Cefoperazone/sulbactam & 0.00 & 12.68 & 19.78 & 8.87 & 0.01 \\
\hline Cefoxitin & 11.76 & 9.83 & 15.12 & 8.20 & 0.02 \\
\hline Aztreonam & 12.87 & 15.03 & 29.59 & 48.07 & 0.00 \\
\hline Amikacin & 0.00 & 3.12 & 5.85 & 13.09 & 0.00 \\
\hline Gentamicin & 1.96 & 4.69 & 7.26 & 6.81 & 0.03 \\
\hline Ciprofloxacin & 0.91 & 6.67 & 11.25 & 8.78 & 0.01 \\
\hline Levofloxacin & 0.00 & 6.07 & 12.96 & 36.32 & 0.00 \\
\hline Macrodantin & 26.73 & 21.90 & 34.54 & 27.28 & 0.00 \\
\hline Sulfamethoxazole & 11.54 & 13.82 & 23.54 & 31.86 & 0.00 \\
\hline Tobramycin & 4.95 & 24.68 & 32.98 & 39.62 & 0.00 \\
\hline Ertapenem & 22.75 & 26.42 & 3.27 & 0.20 \\
\hline Imipenem & 5.00 & 5.49 & 12.54 & 38.44 & 0.00 \\
\hline Meropenem & 13.20 & 7.91 & 1.45 & 0.48 \\
\hline Tigecycline & 0.00 & 18.35 & 6.39 & 0.04 \\
\hline
\end{tabular}


Table 4

Comparison of antibiotic resistance rates (\%) of $K$. pneumoniae from different genders

\begin{tabular}{|c|c|c|c|c|}
\hline Antibiotics & Male & Female & $x^{2}$ & $p$ \\
\hline Ampicillin & 92.52 & 81.53 & 40.91 & 0.00 \\
\hline Cefazolin & 32.76 & 23.63 & 11.37 & 0.00 \\
\hline Ceftazidime & 22.09 & 17.23 & 4.59 & 0.03 \\
\hline Ceftriaxone & 30.57 & 23.50 & 11.73 & 0.00 \\
\hline Cefepime & 17.94 & 9.44 & 27.694 & 0.00 \\
\hline Amoxicillin / Clavulanic acid & 18.36 & 8.05 & 30.18 & 0.00 \\
\hline Piperacillin/Tazobactam & 12.23 & 6.71 & 16.07 & 0.00 \\
\hline Cefoperazone/sulbactam & 18.27 & 13.93 & 2.59 & 0.11 \\
\hline Cefoxitin & 15.06 & 9.40 & 9.90 & 0.00 \\
\hline Aztreonam & 26.93 & 16.57 & 22.97 & 0.00 \\
\hline Amikacin & 5.94 & 2.24 & 15.32 & 0.00 \\
\hline Gentamicin & 19.06 & 12.67 & 10.97 & 0.00 \\
\hline Ciprofloxacin & 32.07 & 24.47 & 10.44 & 0.00 \\
\hline Levofloxacin & 21.10 & 15.78 & 8.74 & 0.00 \\
\hline Macrodantin & 31.80 & 22.29 & 16.55 & 0.00 \\
\hline Sulfamethoxazole & 24.96 & 24.66 & 0.02 & 0.88 \\
\hline Tobramycin & 11.64 & 6.04 & 13.93 & 0.00 \\
\hline Ertapenem & 12.18 & 5.53 & 24.16 & 0.00 \\
\hline Imipenem & 11.69 & 5.09 & 25.22 & 0.00 \\
\hline Meropenem & 8.52 & 3.36 & 4.20 & 0.04 \\
\hline Tigecycline & 7.21 & 3.85 & 7.04 & 0.01 \\
\hline
\end{tabular}

K. pneumoniae drug sensitivity results in different specimens

The resistance rates of $K$. pneumoniae isolated from blood to ertapenem, imipenem, piperacillin/tazobactam, aztreonam, and cefepime were $22.09 \%, 21.59 \%, 40.91 \%, 27.27 \%$, respectively. It was higher than the $K$. pneumoniae isolated from other specimens $(p<0.05)$. The $K$. pneumoniae isolated from urine is significantly higher than the K. pneumoniae isolated from other specimens for levofloxacin, ciprofloxacin, cefoperazone/sulbactam, piperacillin/tazobactam, amikacin, and gentamicin $(p<0.05)$, as shown in Table 5. 
Table 5

Comparison of antibiotic resistance rate (\%) of $K$. pneumoniae in different specimens

\begin{tabular}{|llllllll|}
\hline Antibiotics & $\begin{array}{l}\text { Respiratory } \\
\text { tract samples }\end{array}$ & Urine & Blood & $\begin{array}{l}\text { Sterile } \\
\text { body } \\
\text { fluids }\end{array}$ & $\begin{array}{l}\text { Non-sterile } \\
\text { body fluids }\end{array}$ & X2 & $p$ \\
\hline Ampicillin & 91.88 & 86.07 & 93.85 & 94.63 & 73.17 & 77.48 & 0.00 \\
\hline Cefazolin & 26.65 & 43.09 & 47.73 & 32.74 & 16.54 & 47.09 & 0.00 \\
\hline Ceftazidime & 15.86 & 33.76 & 32.05 & 20.98 & 14.13 & 44.68 & 0.00 \\
\hline Ceftriaxone & 23.49 & 43.71 & 40.23 & 33.91 & 17.16 & 75.73 & 0.00 \\
\hline Cefepime & 12.17 & 24.75 & 27.27 & 19.05 & 4.61 & 69.86 & 0.00 \\
\hline Amoxicillin / Clavulanic & 13.68 & 21.59 & 20.83 & 21.19 & 3.32 & 39.80 & 0.00 \\
\hline acid & & 18.21 & 20.45 & 10.87 & 1.64 & 58.81 & 0.00 \\
\hline Piperacillin/Tazobactam & 8.87 & 32.77 & 21.43 & 18.12 & 17.14 & 28.52 & 0.00 \\
\hline Cefoperazone/sulbactam & 12.88 & 24.43 & 18.75 & 20.53 & 3.32 & 53.31 & 0.00 \\
\hline Cefoxitin & 10.94 & 35.92 & 40.91 & 25.66 & 10.34 & 65.95 & 0.00 \\
\hline Aztreonam & 20.29 & 10.37 & 6.82 & 5.63 & 0.99 & 39.02 & 0.00 \\
\hline Gentamicin & 3.22 & 24.59 & 20.00 & 18.12 & 12.54 & 18.70 & 0.001 \\
\hline Ciprofloxacin & 14.21 & 42.45 & 39.39 & 29.14 & 21.538 & 36.25 & 0.00 \\
\hline Levofloxacin & 25.99 & 32.34 & 27.27 & 25.54 & 10.86 & 68.85 & 0.00 \\
\hline Macrodantin & 15.11 & 32.38 & 40.00 & 34.23 & 16.03 & 30.39 & 0.00 \\
\hline Sulfamethoxazole & 28.09 & 36.88 & 24.14 & 32.17 & 24.34 & 43.79 & 0.00 \\
\hline Tobramycin & 19.82 & 16.33 & 18.18 & 9.93 & 2.43 & 37.71 & 0.00 \\
\hline Ertapenem & 8.49 & 17.45 & 22.09 & 16.38 & 2.64 & 70.24 & 0.00 \\
\hline Imipenem & 7.97 & 14.52 & 22.73 & 12.82 & 2.40 & 56.45 & 0.00 \\
\hline Meropenem & 8.38 & 13.79 & 12.50 & 9.52 & 6.38 & 7.59 & 0.11 \\
\hline Tigecycline & 4.81 & 10.40 & 8.51 & 14.47 & 1.65 & 40.06 & 0.00 \\
\hline
\end{tabular}

Detection rate of ESBL-producing and carbapenem-resistant K. pneumoniae

Among the 1954 strains of K. pneumoniae, the detection rate of CRKP was $10.18 \%$, and the detection rate of ESBL production was $20.97 \%$ ( Fig. 1). The detection rate of ESBL in TCM hospitals was $11.80 \%$, the lowest among all hospitals. The detection rate of CRKP in SMCH is the lowest among all hospitals, only $0.69 \%$. Among all hospitals, the detection rates of ESBL and CRKP in SUH were the highest, which were 33.33\% and $23.37 \%$ respectively. There were significant differences in the detection rates of ESBL and CRKP among all hospitals (ESBL: $\chi 2=30.04, p=0.00$, CRKP: $\chi 2=106.26, p=0.00$ ), as shown in Fig. $1 \mathrm{a}$. The detection rate of ESBLs in the $\leqq 17 y$ group was $23.08 \%$, and no CRKP strain was detected. ESBLs and CRKP in the $\geqq 65 y$ group were $25.47 \%$ 
and $13.94 \%$, respectively, and the detection rate was the highest among the three groups. ESBLs were the lowest in the 18-64y group, which was $15.25 \%$. With the increase of age, the detection rate of CRKP increased

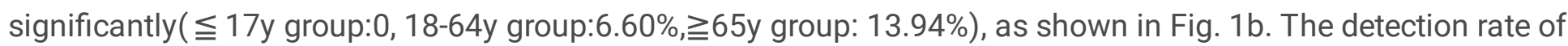
CRKP isolated from males was $13.75 \%$, which was significantly higher than that isolated from females $(7.58 \%)$ $(X 2=18.13, p=0.00)$, as shown in Fig. 1c. The ESBLs rates of respiratory tract specimens, urine, blood, sterile specimens, and non-sterile specimens were $17.50 \%, 32.42 \%, 25.71 \%, 37.37 \%$, and $16.95 \%$, respectively. The CRKP rates were $8.85 \%, 18.48 \%, 23.86 \%, 17.09 \%$, and $2.63 \%$, respectively. as shown in Fig. $1 \mathrm{~d}$.

\section{Discussions}

K. pneumoniae is an opportunistic pathogen that causes a wide range of infections, including pneumonia, urinary tract infection, bacteremia, cholecystitis, and meningitis, especially in immunocompromised individuals. CHINET shows that both the $K$. pneumoniae isolation rate and drug resistance rate are steadily increasing [2]. Data from 66 hospitals in Shanghai show that the separation rate of ESBLs-KP derived from sputum, blood, and urine increased year by year from 2015 to 2017 [9]. The change in antibiotic resistance rates over 17 years (2000-2016) from EARSNet was becoming more and more serious, and that the resistance rates to thirdgeneration cephalosporins, fluoroquinolones, and aminoglycosides showed a significant upward trend, and the analysis was related to ambient temperature [10].

The results of this study show that, except for $\mathrm{SMCH}$, mainly from middle-aged and elderly patients, and with increasing age, the detection rate of $K$. pneumoniae gradually increases; the detection rate of $K$. pneumoniae isolated from men is higher than that of women, and $K$. pneumoniae isolated from males is more resistant to antibiotics than females. These findings suggest that elderly men are facing a more severe situation of $K$. pneumoniae resistance. Population-based surveillance is essential for monitoring the emergence and trends of antimicrobial resistance and guiding the development of rational antibiotic treatment recommendations[11].

Compared with the meta-analysis results of multiple studies in Asia, the overall resistance rates of amikacin, aztreonam, and meropenem were $40.8 \%, 73.3 \%$, and $62.7 \%$, respectively[12], the drug resistance rate in this study area is generally low. In our study, the resistance rate of amikacin was the lowest at $4.42 \%$, followed by tigecycline, meropenem, imipenem, ertapenem, piperacillin/tazobactam, and the resistance rates were 5.76\%, $6.83 \%, 9.42 \%, 9.02 \%, 9.95 \%$, respectively. The resistance rate of the remaining antibiotics is greater than $10 \%$, and the highest resistance rate is ciprofloxacin, with a resistance rate of $28.72 \%$, which was generally lower than the CHINET average[13]. The high resistance rate of ciprofloxacin mainly comes from the urinary tract. The resistance rates of $K$. pneumoniae isolated from urine in this study to ciprofloxacin and levofloxacin were $42.45 \%$ and $32.34 \%$, respectively, which were much higher than those of $K$. pneumoniae isolated from other specimens. This may be because fluoroquinolones are the first-line treatment of empirical medication in urinary tract infections[14, 15].

The production of ESBLs is one of the main resistance mechanisms of $K$. pneumoniae. ESBLs genes are mostly mediated by plasmids, which can spread between bacteria, including TEM, OXA, CTX-M, and SHV, etc, among which CTX-M is the main type contribution of the epidemiologic evolution of human ESBL infections[16]. An epidemiological survey in Zhejiang Province shows that the ESBLs rate is slightly higher than $20 \%$ in recent 
years[17]. The results in 2019 in the Shaoxing region are consistent with it. However, the ESBLs rate of each hospital is different. Among them, the ESBLs rate of TCM Hospital is the lowest, at only $11.80 \%$. Whether it is related to the different disease composition of the patients admitted to this hospital or maybe because Chinese medicine is mainly used and antibiotics are used less is unknown, which is limited study on epidemiology and mechanism in TCM hospitals. A retrospective surveillance study in a TCM hospital in Hangzhou, Zhejiang Province, showed that $11.2 \%$ of hospital-acquired bloodstream infections were caused by $K$. pneumoniae, and $57.1 \%$ stains produced ESBLs, which is significantly higher than the average level of ESBLs in this study[18]. In our study, SUH has the highest ESBLs rate, which was converted from an infectious disease hospital to a general hospital in July 2018. The patients are mainly immunosuppressed people such as liver disease and HIV. The long hospital stay and repeated hospitalization history of these patients may be the main reasons.

K. pneumoniae may have become one of the important pathogens of childhood infections, and also is one of the vital cause of mortality[19]. A systematic review suggests that $K$. pneumoniae is the most frequent etiology of community-acquired pneumonia among children in China[20]. In our study, the detection rate of K. pneumoniae in the $\leqq 17 y$ group was $5.32 \%$, much lower than previous reports[21]. Previous study has shown that children with bloodstream infections have the highest isolation rate and are mainly isolated from the ICU[22]. The $K$. pneumoniae isolated from the $\mathrm{SMCH}$ has the lowest resistance rate to carbapenems, $\beta$-lactam- $\beta$-lactamase inhibitor combinations, fourth-generation cephalosporins, amikacin, tobramycin, and sulfamethoxazole. However, the detection rate of ESBL strains in $\leqq 17 y$ group is $23.08 \%$. Among them, up to $83.33 \%(20 / 24)$ of the strains were isolated from the neonatal ICU of the hospital (data not shown), which was higher than the adult group in this study. Children infected with ESBLs strains had a significantly longer length of hospital stays and required more ICU care days than those without such infection. At the same time, it increases the risk of further acquisition and transmission with resistant bacteria[23]. But the separation rate of ESBL is lower than that of large tertiary children's hospitals in our country[24]. And fortunately, no carbapenem resistance strain was found in $\leqq 17 y$ group in this study.

CHINET surveillance showed that the national average CRKP isolation rate in 2019 was 27.6\%[13]. The 20142018 survey in Zhejiang Province indicated that the CRKP prevalence rate in Shaoxing was $9.72 \%$, the detection rate by our study is slightly higher than that, which is far lower than the national average level[17]. However, the hospitals covered by the above-mentioned research studies are completely different, and there are also certain differences from the six hospitals covered by this research.

The detection rate of CRKP in different specimen sources is varied. In our study, the detection rate in non-sterile body fluids is the lowest at $2.63 \%$, and except for cefoperazone/sulbactam and Sulfamethoxazole, $K$. pneumoniae isolated from non-sterile body fluids has the lowest resistance rate to antibiotics, while the detection rate of CRKP in the blood group is the highest at $23.86 \%$. In our study, the resistance rate of $K$. pneumoniae isolated from blood to imipenem, cefepime, piperacillin/tazobactam is higher than that of strains isolated from other specimen sources. Previous research of bloodstream infections by Yang et al. found that $K$. pneumoniae was the second most infectious pathogen, and both adults and children showed an increasing trend between 2012 and 2017, and resistance to carbapenems and amikacin was on the rise [25]. Bloodstream infections are often combined with infections of other sites, and the most common sites are the respiratory tract and urinary tract[26]. There is also a study that intra-abdominal infection is an important source[27].Carbapenems have been considered to be the last line of defense against $K$. pneumoniae infection. With increasing drug resistance makes 
clinical anti-infective treatment has become a more difficult problem. Especially carbapenem-resistant $K$. pneumoniae bloodstream infections are accompanied by higher mortality[10].

\section{Conclusions}

In brief, the drug resistance situation of $K$. pneumoniae is becoming more and more serious. In different hospitals in the same area and different specimen sources, the clinical distribution and drug resistance of $K$. pneumoniae are very different. Surveillance systems are vital to monitor the emergence and trends in antimicrobial resistance. They are helpful for the development of rational and effective empirical therapy recommendations and policies for limiting the spread of drug-resistant K. pneumoniae. When treating infections caused by $K$. pneumoniae, clinicians should give full consideration to factors such as gender, age, source of specimens, and cross-hospital visits to patients, to rationally use antibiotics. Besides, it is necessary for us to further study the mechanism of $K$. pneumoniae resistance in this region to lay the foundation for better prevention and control.

\section{Abbreviations}

MDR: Multidrug resistance ; CRE: Carbapenem-resistant Enterobacteriaceae; CRKP: Carbapenem-resistant K. pneumoniae ; CHINET: China antimicrobial surveillance network; CRPA: Carbapenem-resistant P. aeruginosa ; ESBLs: Extended-spectrum $\beta$-lactamases; CLSI: Clinical and Laboratory Standards Institute; ATCC: American Type Culture Collection.

\section{Declarations}

\section{Authors' contributions}

GFM conceived and designed the study. XJZ, QLH, QHY, SLW, FXJ, SD, XQL, and LL performed the susceptibility testing and collected data. YCR and MHL analyzed the data. MCL wrote the manuscript. YQZ critically reviewed and edited the manuscript. All coauthors have read, commented, and approved the final version of the article to be published.

\section{Funding}

This work was supported in part by the Shaoxing Science and Technology Plan (grant 2018C30101 and 2020A13029). and the Zhejiang Health Science and Technology Project (grant 2021KY1138 and 2021RC134).

\section{Availability of data and materials}

The data and materials used and analyzed during the current study are available from the corresponding author on reasonable request.

\section{Ethics approval and consent to participate}

Ethical approval was approved by the Institutional Review Board of Shaoxing People's Hospital, who waived the need for informed consent. The study is conducted on already available data. All inpatients data were anonymized before analysis. Acquisition of participating hospital data was approved by the scientific research department of the hospital. 


\section{Consent for publication}

Not applicable.

\section{Conflicts of interest}

The authors declare that they have no conflict of interest.

\section{Author details}

${ }^{1}$ Department of Clinical Laboratory, Shaoxing People's Hospital (Shaoxing Hospital, Zhejiang University School of Medicine), Zhongxing Road 566, Shaoxing 312000, Zhejiang Province, People's Republic of China.

2 Department of Clinical Laboratory, Shaoxing Maternity and Child Health Care Hospital, Dongjie 305, Shaoxing 312000, Zhejiang Province, People's Republic of China.

3 Department of Clinical Laboratory, Shaoxing Second Hospital, East Yan'an Road 123, Shaoxing 312000, Zhejiang Province, People's Republic of China.

${ }^{4}$ Department of Clinical Laboratory, The Affiliated Hospital of Shaoxing University, South Zhongxing Road 999, Shaoxing 312000, Zhejiang Province, People's Republic of China.

${ }^{5}$ Department of Clinical Laboratory, Shaoxing Traditional Chinese Medicine Hospital, Renmin Road 641, Shaoxing 312000, Zhejiang Province, People's Republic of China.

${ }^{6}$ Department of Clinical Laboratory, Shaoxing Seventh Hospital, West Shengli Road 1234, Shaoxing 312000, Zhejiang Province, People's Republic of China.

7 Department of Infectious Diseases, Shaoxing People's Hospital (Shaoxing Hospital, Zhejiang University School of Medicine), Zhongxing Road 566, Shaoxing 312000, Zhejiang Province, People's Republic of China.

\section{References}

1. Zhang Y, Wang Q, Yin Y, Chen H, Jin L, Gu B, et al. Epidemiology of Carbapenem-resistant Enterobacteriaceae infections: Report from the China CRE Network. Antimicrob Agents Chemother. 2018;62(2):e01882-17.

2. Hu F, Guo Y, Yang Y, Zheng Y, Wu S, Jiang X, et al. Resistance reported from China antimicrobial surveillance network (CHINET) in 2018. Eur J Clin Microbiol Infect Dis. 2019;38(12):2275-81.

3. World Health Organization. Global priority list of antibiotic-resistant bacteria to guide research,discovery, and development of new antibiotics.. 2017. [cited 2020 Nov 15]. Available from: https://www.who.int/medicines/publications/WHO-PPL-Short_Summary_25Feb-ET_NM_WHO.pdf.

4. Zhen X, Stålsby Lundborg C, Sun X, Gu S, Dong H. Clinical and economic burden of Carbapenem-resistant infection or colonization caused by Klebsiella pneumoniae, Pseudomonas aeruginosa, Acinetobacter baumannii: A Multicenter Study in China. Antibiotics (Basel). 2020;9(8):514.

5. Partridge SR, Kwong SM, Firth N, Jensen SO. Mobile genetic elements associated with antimicrobial resistance. Clin Microbiol Rev. 2018;31(4):e00088-17. 
6. Han R, Shi Q, Wu S, Yin D, Peng M, Dong D, et al. Dissemination of Carbapenemases (KPC, NDM, OXA-48, IMP, and VIM) among carbapenem-resistant Enterobacteriaceae isolated from adult and children patients in China. Front Cell Infect Microbiol. 2020;10:314.

7. Clinical and Laboratory Standards Institute. Performance standards for antimicrobial susceptibility testing, M100 28th edition:Wayne, PA,2018.

8. Centers for Disease Control and Prevention. Facility guidance for control of carbapenem-resistant Enterobacteriaceae (CRE)-November 2015 Update CRE Toolkit.2015. [cited 2020 Nov 15]. Available from:https://www.cdc.gov/hai/pdfs/cre/CRE-guidance-508.pdf.

9. Lin J, Gao X, Cui Y, Sun W, Shen Y, Shi Q, et al. Increased multidrug resistant isolates: new clinical burdens for 66 hospitals in Shanghai, 2015 to 2017. Ann Transl Med. 2020;8(4):112.

10. McGough SF, MacFadden DR, Hattab MW, Mølbak K, Santillana M. Rates of increase of antibiotic resistance and ambient temperature in Europe: a cross-national analysis of 28 countries between 2000 and 2016 . Euro Surveill. 2020;25(45):pii = 1900414.

11. Sawatwong P, Sapchookul P, Whistler T, Gregory CJ, Sangwichian O, Makprasert S, et al. High burden of extended-spectrum $\beta$-Lactamase-producing Escherichia coli and Klebsiella pneumoniae bacteremia in older adults: A seven-year study in two rural Thai provinces. Am J Trop Med Hyg. 2019;100(4):943-51.

12. Effah CY, Sun T, Liu S, Wu Y. Klebsiella pneumoniae: an increasing threat to public health. Ann Clin Microbiol Antimicrob. 2020;19(1):1.

13. Hu FP, Guo Y, Zhu DM, Wang F, Jiang XF, Xu YC, et al. CHINET surveillance of bacterial resistance across tertiary hospitals in 2019. Chin J Infect Chemother. 2020;20(03):233-43.

14. Schaeffer AJ, Nicolle LE. Urinary tract infections in older men. N Engl J Med. 2016;374(6):562-71.

15. Caron F, Galperine T, Flateau C, Azria R, Bonacorsi S, Bruyère F, et al. Practice guidelines for the management of adult community-acquired urinary tract infections. Med Mal Infect. 2018;48(5):327-58.

16. Adler A, Katz DE, Marchaim D. The continuing plague of extended-spectrum $\beta$-lactamase producing Enterbacterales infections: An update. Infect Dis Clin North Am. 2020;34(4):677-708.

17. Hu Y, Liu C, Shen Z, Zhou H, Cao J, Chen S, et al. Prevalence, risk factors and molecular epidemiology of carbapenem-resistant Klebsiella pneumoniae in patients from Zhejiang, China, 2008-2018. Emerg Microbes Infect. 2020;9(1):1771-9.

18. Al-Flahi A, Zou JK, Yin XF. Solid-phase microextraction for flavor analysis in Harari Khat (Catha edulis) stimulant. J Zhejiang Univ Sci. 2004;5(4):428-31.

19. Taylor AW, Blau DM, Bassat Q, Onyango D, Kotloff KL, Arifeen SE, et al. Initial findings from a novel population-based child mortality surveillance approach: a descriptive study. Lancet Glob Health. 2020;8(7):e909-19.

20. Ning G, Wang X, Wu D, Yin Z, Li Y, Wang H, et al. The etiology of community-acquired pneumonia among children under 5 years of age in mainland China, 2001-2015: A systematic review. Hum Vaccin Immunother. 2017;13(11):2742-50.

21. Hu YJ, Ogyu A, Cowling BJ, Fukuda K, Pang HH. Available evidence of antibiotic resistance from extendedspectrum $\beta$-lactamase-producing Enterobacteriaceae in paediatric patients in 20 countries: a systematic review and meta-analysis. Bull World Health Organ. 2019;97(7):486-501B. 
22. Mamishi S, Mahmoudi S, Naserzadeh N, Hosseinpour Sadeghi R, Haghi Ashtiani MT, Bahador A, et al. Antibiotic resistance and genotyping of gram-negative bacteria causing hospital-acquired infection in patients referred to Children's Medical Center. Infect Drug Resist. 2019;12:3377-84.

23. Badal RE, Bouchillon SK, Lob SH, Hackel MA, Hawser SP, Hoban DJ. Etiology, extended-spectrum $\beta$ lactamase rates and antimicrobial susceptibility of gram-negative bacilli causing intra-abdominal infections in patients in general pediatric and pediatric intensive care units-global data from the Study for Monitoring Antimicrobial Resistance Trends 2008 to 2010. Pediatr Infect Dis J. 2013;32(6):636-40.

24. Li X, Ding X, Shi P, Zhu Y, Huang Y, Li Q, et al. Clinical features and antimicrobial susceptibility profiles of culture-proven neonatal sepsis in a tertiary children's hospital, 2013 to 2017. Medicine. 2019;98(12):e14686.

25. Yang S, Xu H, Sun J, Sun S. Shifting trends and age distribution of ESKAPEEc resistance in bloodstream infection, Southwest China, 2012-2017. Antimicrob Resist Infect Control. 2019;8:61.

26. Li Y, Li J, Hu T, Hu J, Song N, Zhang Y, et al. Five-year change of prevalence and risk factors for infection and mortality of carbapenem-resistant Klebsiella pneumoniae bloodstream infection in a tertiary hospital in North China. Antimicrob Resist Infect Control. 2020;9(1):79.

27. Imai K, Ishibashi N, Kodana M, Tarumoto N, Sakai J, Kawamura T, et al. Clinical characteristics in blood stream infections caused by Klebsiella pneumoniae, Klebsiella variicola, and Klebsiella quasipneumoniae: a comparative study, Japan, 2014-2017. BMC Infect Dis. 2019;19(1):946.

\section{Figures}




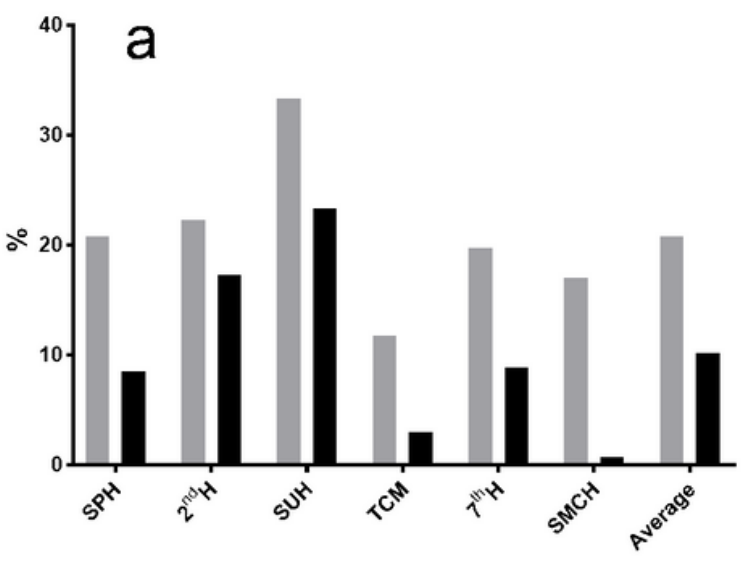

Hosptials

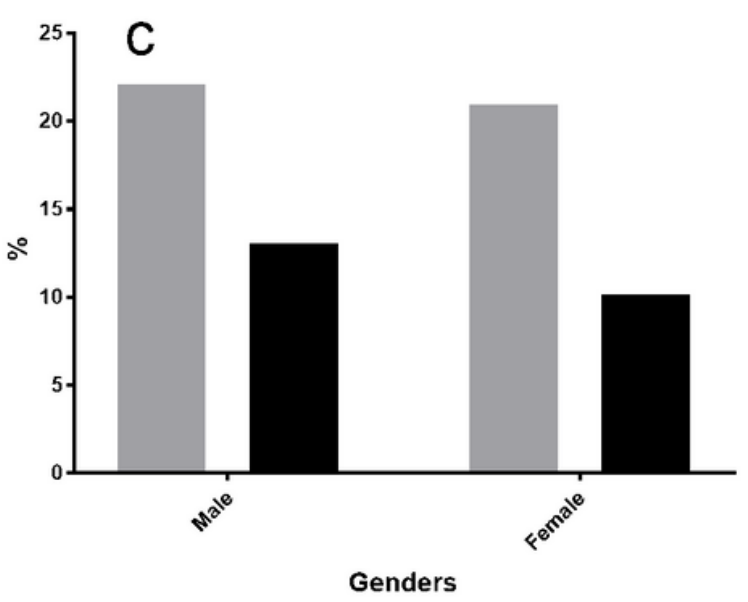

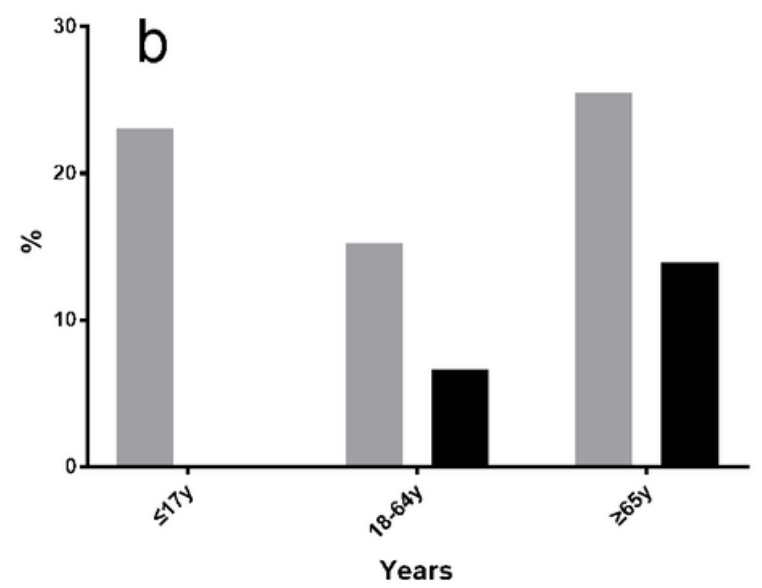

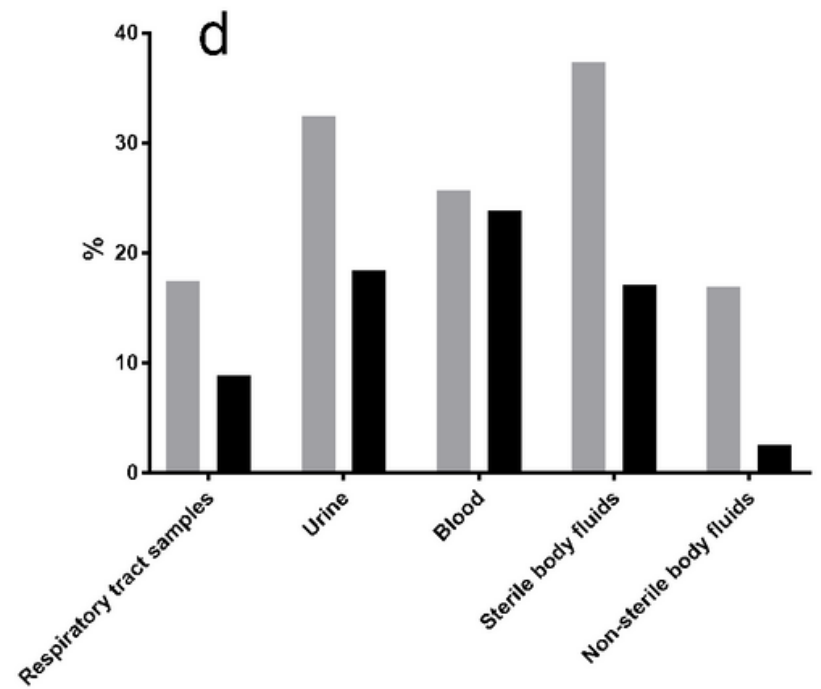

Specimens

Figure 1

ESBL-KP and CR-KP distribution ESBL rate (gray) and CR rate (black) of all strains. Among them, Fig.1a shows the ESBL rate and CR rate of strains isolated from different hospitals; Fig. 1b shows the ESBL rate and CR rate of strains from patients with different age groups; Fig.1c shows the ESBL rate and CR rate of strains from dif-ferent genders; and Fig.1d shows the ESBL rate and CR rate of strains from different specimens. 